\title{
Parametric evaluation of part distortion in additive manufacturing processes
}

\author{
Giacomo Quaranta ${ }^{1}$. Eberhard Haug ${ }^{1} \cdot$ Jean Louis Duval ${ }^{1} \cdot$ Francisco Chinesta $^{2}$ (I)
}

\begin{abstract}
Additive manufacturing is the more and more considered in industry, however efficient simulation tools able to perform accurate predictions are still quite limited. The main difficulties for an efficient simulation are related to the multiple scales, the multiple and complex physics involved, as well as the strong dependency on the process trajectory. This paper aims at proposing a simplified parametric modeling and its subsequent parametric solution for evaluating parametrically the manufactured part distortion. The involved parameter are the ones parametrizing the process trajectories, the thermal shrinkage intensity and anisotropy (the former depending on several material and process parameters and the last directly depending on the process trajectory) and the deposited layers. The resulting simulation tool allows evaluating in real-time the impact of the parameters just referred on the part distortion, and proceed to the required geometrical compensation.
\end{abstract}

Keywords Vademecum · Additive manufacturing $\cdot$ PGD $\cdot$ MOR $\cdot$ Thermal shrinkage $\cdot$ Part distortions $\cdot$ Geometry compensation

\section{Introduction}

The adoption of Additive Manufacturing (AM) technologies at the industrial level has set out several challenges for the scientific community, ranging from the study of the interactions at the scale of the particle to the prediction of the mechanical state of the final part $[11,17]$.

Additive manufacturing processes have been extensively simulated by using the Finite Element Method (FEM). In [22] a model for direct laser metal powder deposition process and

Francisco Chinesta

Francisco.Chinesta@ensam.eu

Giacomo Quaranta

Giacomo.Quaranta@esi-group.com

Eberhard Haug

Eberhard.Haug@esi-group.com

Jean Louis Duval

Jean-Louis.Duval@esi-group.com

1 ESI Group, Parc Icade, Immeuble le Séville, 3 bis, Saarinen, CP 50229, 94528, Rungis Cedex, France

2 PIMM, ENSAM ParisTech, ESI GROUP Chair on Advanced Modeling and Simulation of Manufacturing Processes, 151 Boulevard de l'Hôpital, F-75013 Paris, France rapid prototyping was developed and simulated. In [20] and [14] 3D FEM simulations of a thermal model of selective laser sintering were performed for evaluating the temperature evolution. In [26] a thermo-structural finite element analysis of direct laser metal deposited was proposed for evaluating residual stresses in thin-walled structures. In [10] the FEM was considered for simulating shaped metal deposition processes. In [25] a finite element analysis was carried out to compute the temperature induced in aero-engine component by the direct laser deposition process and the corresponding distortion and in [24] and [23] the effects of laser power and scan speed on the thermal behavior were investigated. FEM was also used in many other works, as for example in [16] to develop a thermo-mechanical model of additive manufacturing of Ti-6Al-4V.

Other techniques have also been considered as for instance Lattice Boltzmann [18, 19], Finite Differences [15], Finite Volumes in [3, 12] or discrete models [21] for the processes involving selective laser melting of powders.

A global process modeling and its associated efficient solution remain nowadays out of reach because many challenges persist, concerning the multiple physics and scales requiring fine and rich models, well calibrated in extreme conditions (in space, time and process conditions). These circumstances and the extremely long process trajectory length compromise the efficiency of well 
experienced numerical simulations tools. These difficulties can be grouped in three categories:

- Geometric complexity. The flexibility of AM technologies allows for the use of topology optimization in the design stage. This results typically in thin parts, very sensitive to distortions. Complex geometries also require finer meshes and hence, large size numerical models.

- Strong dependence on the fabrication strategy. This is mainly due to the non-linear and coupled models involved in phase transformations and inelastic behaviors.

- The incremental nature of the process. A part is composed of thousands of deposited layers each of them constituted of a succession of tapes less than $1 \mathrm{~mm}$ thick.

These difficulties justify the fact that standard approaches (e.g. finite elements combined with the so-called born-dead-elements technique [13]) lead to prohibitive simulation times. Thus, well experienced simulation technologies cannot be used for performing parametric analysis and efficiently exploring the parametric design space for optimizing processes or for performing simulation-based real-time control.

Important efforts are being deployed in order to propose new computational strategies. Among them, mesh adaptivity approaches [27], performing refining/coarsening, are widely considered to resolve the region in which the spot applies. However, and despite the significant progresses in mesh adaptation, remeshing entails its own difficulties and motivated in the last decade the proposal and development of meshless methods, that solved some issues and created many others. In any case, coarsening is limited by field localization as well as by the necessity to accurately describe extremely complex geometries.

However, one of the main obstacles to an efficient simulation of AM processes for predicting part distortions, independently of the model richness, is related to the numerical model itself, by the fact of assembling and solving very large systems of equations at each time step and in a geometry that is evolving in time. Thus, reduced order modeling [9] was envisaged as a possible remedy for attaining faster simulations without compromising the accuracy.

Simplified models were proposed by observing that in many cases two regions can be differentiated [1]. The first, near the spot and involving the most recent deposited layers involves rich nonlinear behaviors, however outside, plastic deformation becomes frozen and does not evolve anymore. These regions were called respectively process and structure regions. This partition makes possible the use of static condensation, such that all the degrees of freedom in the structure region are condensed on the interface between both regions. However, such a condensation requires, even in the linear case, the inversion of a sub-matrix (the one related to the structure region) whose size is increasing with the number of deposited layers, and consequently with the growing of the structure region. In [1] authors proposed solving the process by assuming in the structure region a linear elastic behavior with the residual stresses installed when each point now in the structure region was located in the process zone (during its deposition). Thus, after evaluating the residual stress everywhere in the part at the end of the process, a final calculation is performed in the finite transformation framework (assuming that larger deformations / displacements do not alter the installed residual stresses). However, such a calculation allows only evaluating the part distortion but not the distortion evolution during the process.

The present work aims at proposing a simplified parametric modeling and its subsequent parametric solution for evaluating parametrically the manufactured part distortion. The involved parameters are the ones parametrizing the process trajectories, the thermal shrinkage intensity and anisotropy (the former depending on several material and process parameters and the last directly depending on the process trajectory) and the deposited layers. The resulting simulation tool allows evaluating in real-time the impact of the parameters just referred to the part distortion, and proceed to the required geometry compensation.

\section{Model order reduction}

One could think that computing time issues could be circumvented with the mere use of more powerful computers. Even if it is a valuable route, it compromises the accessibility to the appropriate simulation resources of small and medium industries as well as its integration in deployed systems. Model Order Reduction -MOR techniques opened new possibilities for more efficient simulations.

A possible alternative within the MOR framework consists in extracting "offline" the most significant modes involved in the model solution, that will constitute the socalled reduced approximation basis, and then project the solution of "quite similar" problems in that reduced basis. Because the number of functions involved in the reduced basis is quite low, only few coefficients must be calculated for determining the problem solution. Consequently, a discrete problem of very small size must be solved at each iteration or time step. Thus, MOR-based discretization techniques allow important computing-time savings, of several orders of magnitude in some cases, making possible accommodating to real-time constraints [7,9]

Another MOR-based route, close to the previous one, consists of calculating offline using all the needed computational resources, e.g. HPC, and computing time, a 
parametric solution containing the solution of all possible scenarios, that is then particularized online using light computational facilities, as deployed devices, tablets or even smartphones, for performing efficient simulation, optimization, inverse analysis, uncertainty propagation and simulation-based control, all them under real-time constraints.

This was the solution retained in many applications where the so-called PGD - Proper Generalized Decomposition - was able to compute parametric solutions of models representing complex processes, structures and systems [2, $4-6,8,9]$. Thus, few hours of intensive offline computation allow the construction of very general parametric solutions able to ensure almost instantaneous responses to queries, while keeping the level of accuracy of usual discretizations.

Space-time-parameters separated representation allowed constructing the so-called computational vademecums (also known as abacus, virtual charts, nomograms, ...), efficiently considered for multiple purposes: simulation, optimization, inverse analysis, uncertainty propagation and simulationbased control, all them under the real-time constraint [5].

Thus, when the unknown field involves space, time and a series of parameters $\mu_{1}, \ldots, \mu_{\mathrm{Q}}$, its associated separated representation reads

$u\left(\boldsymbol{x}, t, \mu_{1}, \ldots, \mu_{Q}\right) \approx \sum_{i=1}^{\mathrm{M}} X_{i}(\boldsymbol{x}) T_{i}(t) \prod_{j=1}^{Q} M_{i}^{j}\left(\mu_{j}\right)$.

The construction of such separated representations or computational vademecums was deeply addressed in numerous of our former works, and in particular in the primer [6]. The interested reader can also refer to the numerous references therein.

\section{Parametrizing trajectories}

Additive Manufacturing is an incremental process requiring the definition of the process trajectory. In order to efficiently evaluate the impact of the different possible trajectories on the part distortion, for optimizing or controlling the process, one should be able to simulate in almost real-time the process, possibility that remains nowadays out of reach, or creating offline the parametric solution of the process, that is, the output of interest, the part distortion in our case, for any possible process trajectory. Thus, the expensive offline calculation (the vademecum construction) will be largely compensated by its online use in almost real-time, because only particularizations of the parametric solutions are required.

In our former works a panoply of different type parameters were introduced as extra-coordinates in the model,

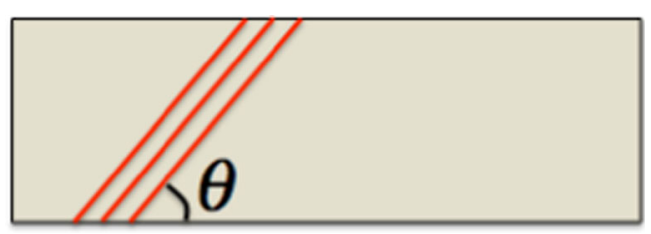

Fig. 1 One-parameter process trajectories

leading to a parametric solution like (1). In that expression parameters $\mu_{i}$ can be material parameters, process parameters (including initial and boundary conditions) and even parameters related to the geometry.

Sometimes parameters are not discrete but continuous, for example material parameters can evolve in space and/or time. In that case the solution retained was assuming an appropriate parametrization of these fields, and then including the coefficients of those approximations as model parameters and consequently within the PGD rationale, as problem extra-coordinates.

This option was possible when the field describing the space and/or time evolution of the parameters involved few terms, however in the case of a trajectory the situation is radically different because any two points $\boldsymbol{P}$ and $\boldsymbol{Q}$ and independently on the distance between them, can be joined by an infinity of curves, and then parametrizing a trajectory has no sense. Thus, rather than looking for all possible trajectories, one must restrict to a family of them.

To fix ideas we first consider the additive manufacturing process of a solid cuboid shape and the addition of a new rectangular layer on its top surface. A family of trajectories for covering all its surface could consists of straight lines parametrized by a single parameter, e.g. the angle $\theta$ with respect to the rectangle base, as depicted in Fig. 1. However, such a parametrization does not allow other usual process trajectories as the one illustrated in Fig. 2.

A general approach consists in associating the trajectories to the iso-field curves $\phi=$ cte related to a certain parametrized partial differential equation involving the scalar field $\phi(x, y)$. For that purpose we propose considering the Poisson equation defined in the rectangular domain $\Omega=(0, L) \times(0, H)$

$\Delta \phi(x, y)=s(x, y)$,

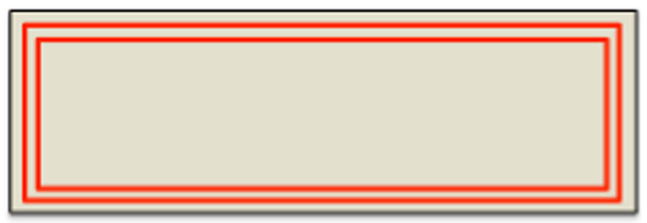

Fig. 2 Concentric-like trajectories 

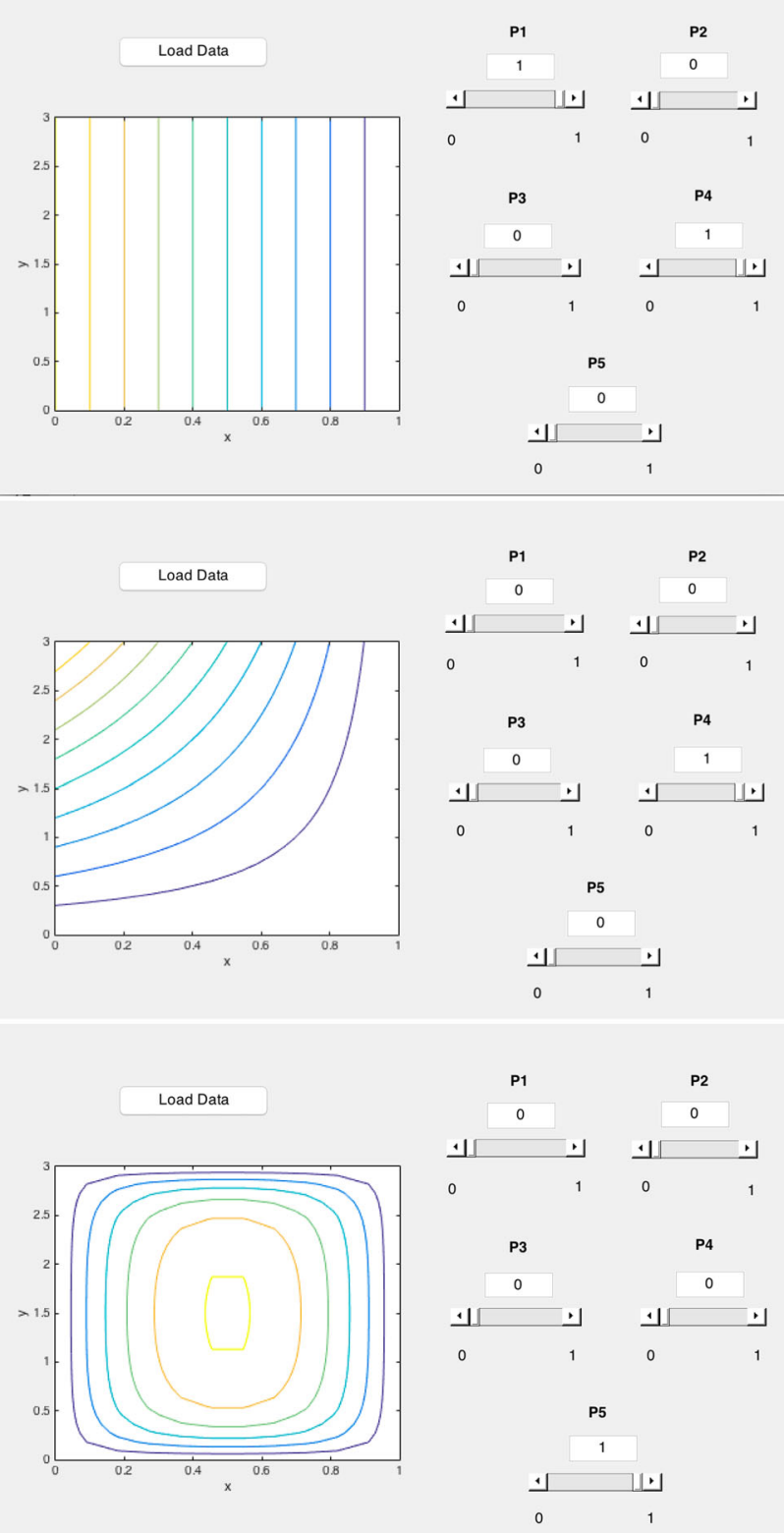

Fig. 3 Trajectories for: (top-left) $\mu_{1}=1, \mu_{2}=0, \mu_{3}=0, \mu_{4}=$ $1, \mu_{5}=0$; (top-right) $\mu_{1}=1, \mu_{2}=1, \mu_{3}=0, \mu_{4}=0, \mu_{5}=0$; (middle-left) $\mu_{1}=0, \mu_{2}=0, \mu_{3}=0, \mu_{4}=1, \mu_{5}=0$; (middle-right) $\mu_{1}=0.33, \mu_{2}=0, \mu_{3}=0.66, \mu_{4}=1, \mu_{5}=0$;

with the parametrized Dirichlet boundary conditions

$\left\{\begin{array}{l}\phi(x, y=0)=\mu_{1}+\left(\mu_{2}-\mu_{1}\right) \frac{x}{L} \\ \phi(x, y=H)=\mu_{4}+\left(\mu_{4}-\mu_{3}\right) \frac{x}{L} \\ \phi(x=0, y)=\mu_{1}+\left(\mu_{4}-\mu_{1}\right) \frac{y}{H} \\ \phi(x=L, y)=\mu_{2}+\left(\mu_{3}-\mu_{2}\right) \frac{y}{H}\end{array}\right.$,

that represent linear variations on each rectangle side, parametrized by the values at the corners $\phi(0,0)=\mu_{1}$, $\phi(L, 0)=\mu_{2}, \phi(L, H)=\mu_{3}$ and $\phi(0, H)=\mu_{4}$. When all these parameters defining the boundary condition vanish, and the source term takes a unit value, i.e. $s(x, y)=1$,
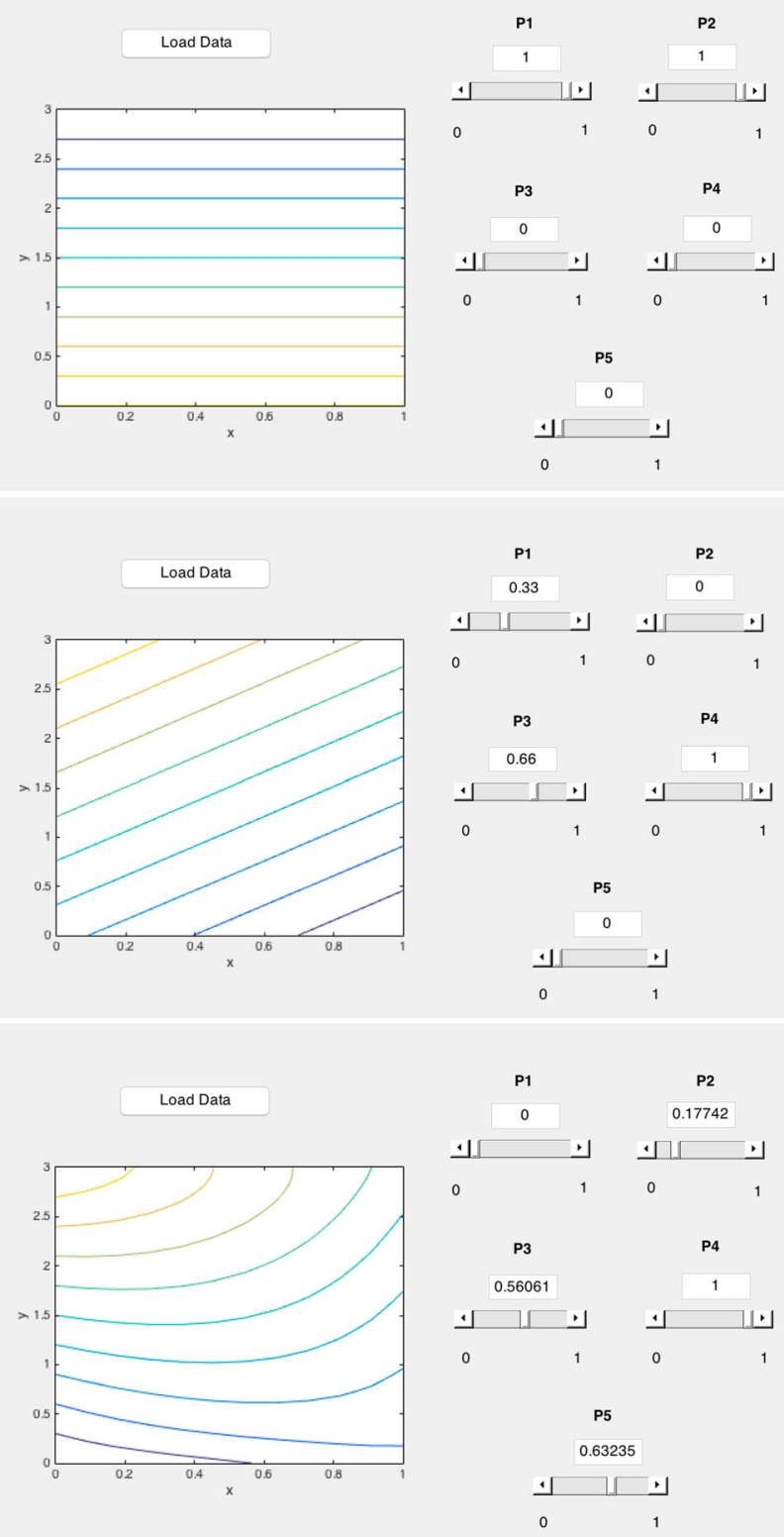

(bottom-left) $\mu_{1}=0, \mu_{2}=0, \mu_{3}=0, \mu_{4}=0, \mu_{5}=1$ and (bottom-right) $\mu_{1}=0, \mu_{2}=0.2, \mu_{3}=0.5, \mu_{4}=1, \mu_{5}=0.6$. Dimensions: $\times 10 \mathrm{~cm}$

the solution iso-curves are expected representing concentric trajectories.

The parametric solution of this problem is easily computable because of its linearity, and consequently the use of the standard constructor of PGD parametric solutions is not compulsory. One must simply solve the following five boundary value problems:

$$
\Delta \phi^{1}(x, y)=s(x, y), \text { with } s(x, y)=0
$$

$\& \mu_{1}=1, \mu_{2}=\mu_{3}=\mu_{4}=0$, 


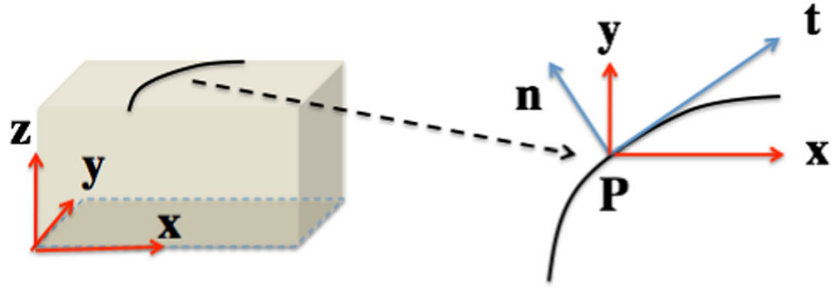

Fig. 4 Wired model for the installed residual stresses

$$
\begin{aligned}
& \Delta \phi^{2}(x, y)=s(x, y), \text { with } s(x, y)=0 \\
& \& \mu_{2}=1, \mu_{1}=\mu_{3}=\mu_{4}=0, \\
& \Delta \phi^{3}(x, y)=s(x, y), \text { with } s(x, y)=0 \\
& \& \mu_{3}=1, \mu_{1}=\mu_{2}=\mu_{4}=0, \\
& \Delta \phi^{4}(x, y)=s(x, y), \text { with } s(x, y)=0 \\
& \& \mu_{4}=1, \mu_{1}=\mu_{2}=\mu_{3}=0,
\end{aligned}
$$

and

$$
\begin{aligned}
& \Delta \phi^{5}(x, y)=s(x, y), \text { with } s(x, y)=1 \\
& \& \mu_{1}=\mu_{2}=\mu_{3}=\mu_{4}=0 .
\end{aligned}
$$

Now, any other solution for any choice of parameters $\mu_{1}, \cdots, \mu_{4}$ and $\mu_{5}=s(x, y)$ can be obtained from

$\phi\left(x, y, \mu_{1}, \cdots, \mu_{5}\right)=\mu_{1} \phi^{1}(x, y)+\cdots+\mu_{5} \phi^{5}(x, y)$.

Obviously richer parametrizations of the boundary conditions as well as of the source term can be envisaged. Even other linear and nonlinear parametrized partial differential equations could be adopted, where in the nonlinear case, where superposition fails, the usual greedy PGD constructor should be considered for circumventing the curse of dimensionality.

Figure 3 depicts different scenarios, where parameters $\mu_{1}, \cdots, \mu_{4}$ and $\mu_{5}=s(x, y)$ are noted by $P 1, \cdots, P 5$. The developed GUI application allows evaluating any possible trajectory related to the problem defined above in almost real-time.

\section{A simplified parametric thermo-mechanical model}

In this section we address parametrized thermal shrinkage. Many simplified AM simulators consider that mechanism as soon as a layer or several layers are assumed deposited at a certain temperature, from which the cooling process occurs, and being the thermal shrinkage constrained by the already solid part, residuals stress are induced.

In this work, among the numerous possibilities we are assuming a wired model that considers that thermal shrinkage follows the deposition trajectory as sketched in Fig. 4.

Thus, in the local system of coordinates, shrinkage stresses are expected having the expression

$\tilde{\boldsymbol{\sigma}}_{0}(\mathbf{P})=\left(\begin{array}{lll}\lambda & 0 & 0 \\ 0 & 0 & 0 \\ 0 & 0 & 0\end{array}\right)$.

It is important to note that when the wired hypothesis is expected working inappropriately, one could consider a richer parametrization of the shrinkage stress tensor, coming from an appropriate micro-to-macro upscaling modeling, and assuming in the most general case an expression containing six parameters (the six components of the symmetric shrinkage stress tensor). In the general case the stress tensor could also depend on the space coordinates. These spatial dependence will result from a micro-mechanical analysis and will require a richer parametric expression by invoking the PGD rationale.

The global equilibrium requires expressing shrinkage stresses, given at each point by expression (10) in the local coordinate system related to the local trajectory, in the global coordinate systems $(x, y, z)$ depicted in Fig. 4. For that purpose we should consider the rotation of it according to

$\boldsymbol{\sigma}_{0}(\mathbf{P})=\mathbf{R}^{T} \tilde{\boldsymbol{\sigma}}_{0}(\mathbf{P}) \mathbf{R}$,

with $\mathbf{R}$ the rotation matrix.

It is expected that when considering the model just described, shrinkage stresses depend parametrically on the trajectory (that controls its anisotropy) and also eventually on space from thermal couplings here ignored.

One could imagine the possibility of expressing the shrinkage tensor parametrically as was the case for the trajectories. Thus, by noting $\boldsymbol{\sigma}^{i}(\mathbf{P})$ the shrinkage tensor related to the parameters choice $\mu_{j}=\delta_{i j}$, with $\delta$ the

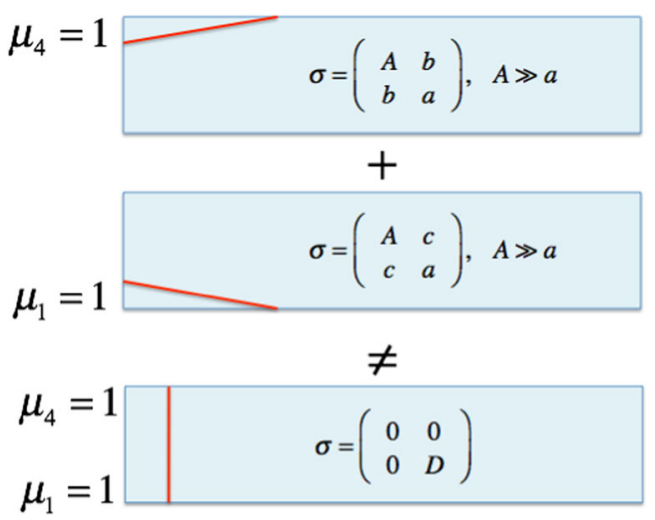

Fig. 5 Shrinkage tensor interpolation issues 


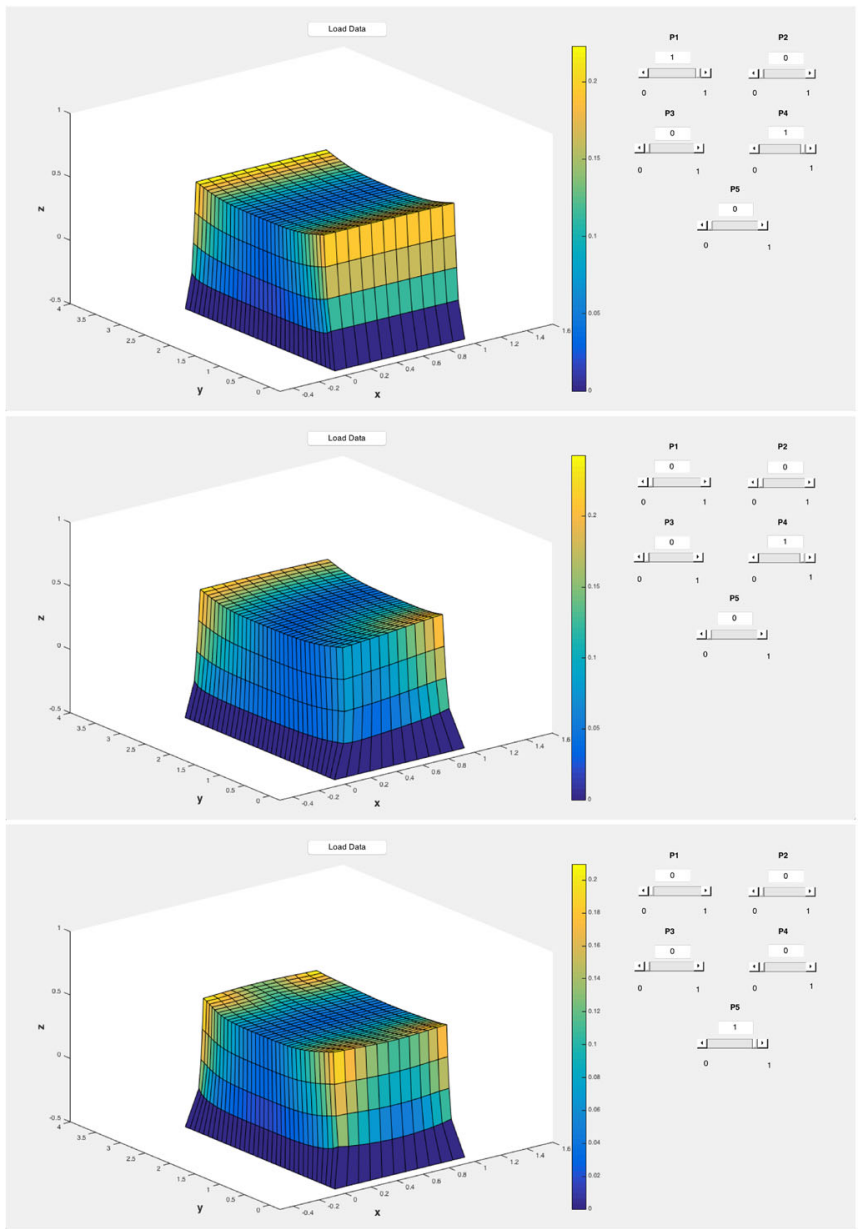

Fig. 6 Parametric distortions for: (top-left) $\mu_{1}=1, \mu_{2}=0, \mu_{3}=$ $0, \mu_{4}=1, \mu_{5}=0$; (top-right) $\mu_{1}=1, \mu_{2}=1, \mu_{3}=0, \mu_{4}=$ $0, \mu_{5}=0$; (middle-left) $\mu_{1}=0, \mu_{2}=0, \mu_{3}=0, \mu_{4}=1, \mu_{5}=0$; (middle-right) $\mu_{1}=0.33, \mu_{2}=0, \mu_{3}=0.66, \mu_{4}=1, \mu_{5}=0$;

Kroneker delta, the thermal shrinkage tensor could be expected expressing as

$$
\sigma_{0}\left(\mathbf{P} ; \mu_{1}, \cdots, \mu_{5}\right)=\mu_{1} \sigma^{1}(\mathbf{P})+\cdots+\mu_{5} \boldsymbol{\sigma}^{5}(\mathbf{P}) .
$$

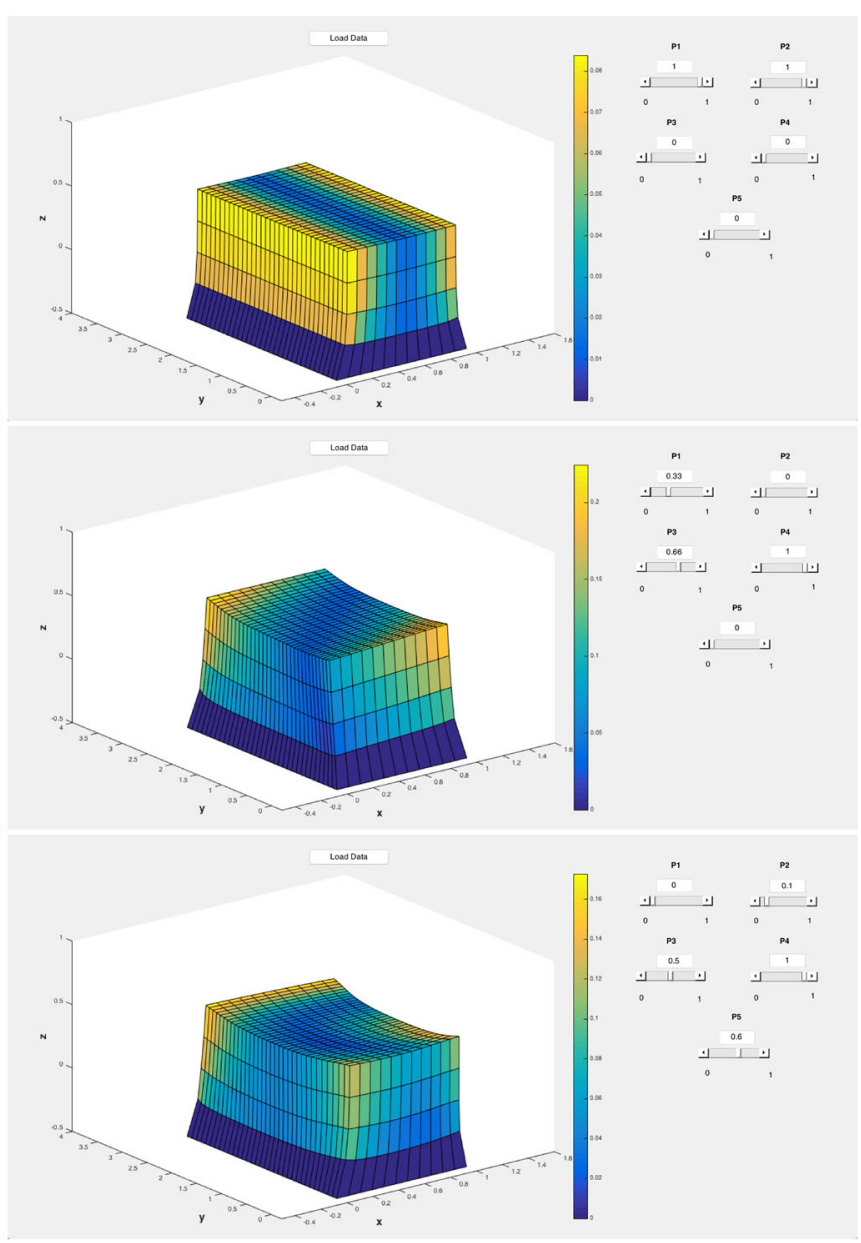

(bottom-left) $\mu_{1}=0, \mu_{2}=0, \mu_{3}=0, \mu_{4}=0, \mu_{5}=1$ and (bottom-right) $\mu_{1}=0, \mu_{2}=0.1, \mu_{3}=0.5, \mu_{4}=1, \mu_{5}=0.6$. The depicted mesh is only for visualization purposes, the one related to the calculations is finer. Dimensions: $\times 10 \mathrm{~cm}$. Displacements in $\mathrm{cm}$

However, such a natural option fails because the limitations of tensor interpolations as Fig. 5 illustrates.

For circumventing the just mentioned difficulties we proceed by expressing the rotation matrix parametrically.
Fig. 7 Distorted geometry for the indicated parameters defining the process trajectory.

Dimensions: $\times 10 \mathrm{~cm}$. Displacements in $\mathrm{cm}$
Deformed shape: $\mu_{1}=0.54688, \mu_{2}=0.95751, \mu_{3}=0.96489, \mu_{4}=0.15761, \mu_{5}=0.97059$

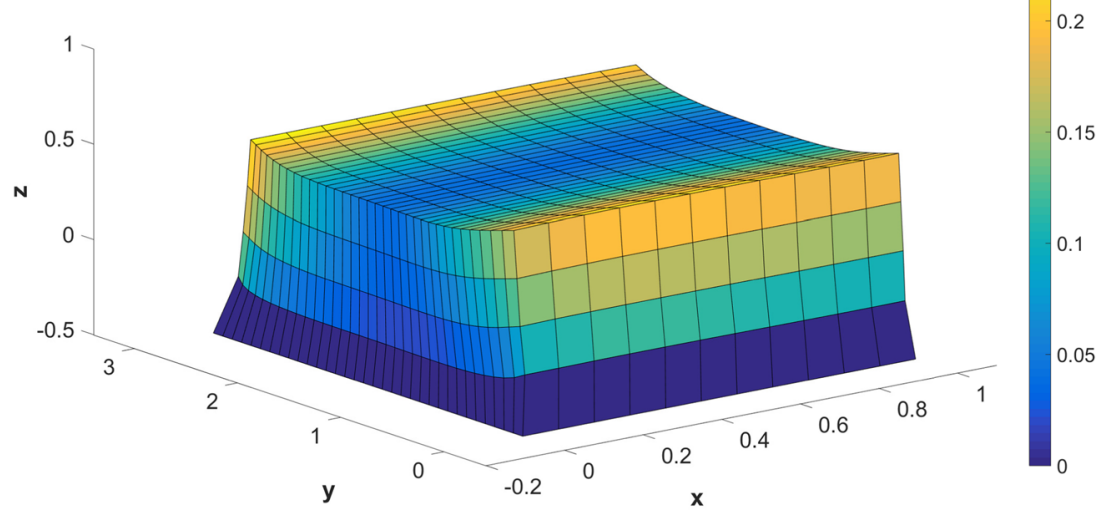


Fig. 8 Compensated geometry.

Dimensions: $\times 10 \mathrm{~cm}$.

Displacements in $\mathrm{cm}$

Fig. 9 Distortion applying on the compensated geometry.

Dimensions: $\times 10 \mathrm{~cm}$.

Displacements in $\mathrm{cm}$
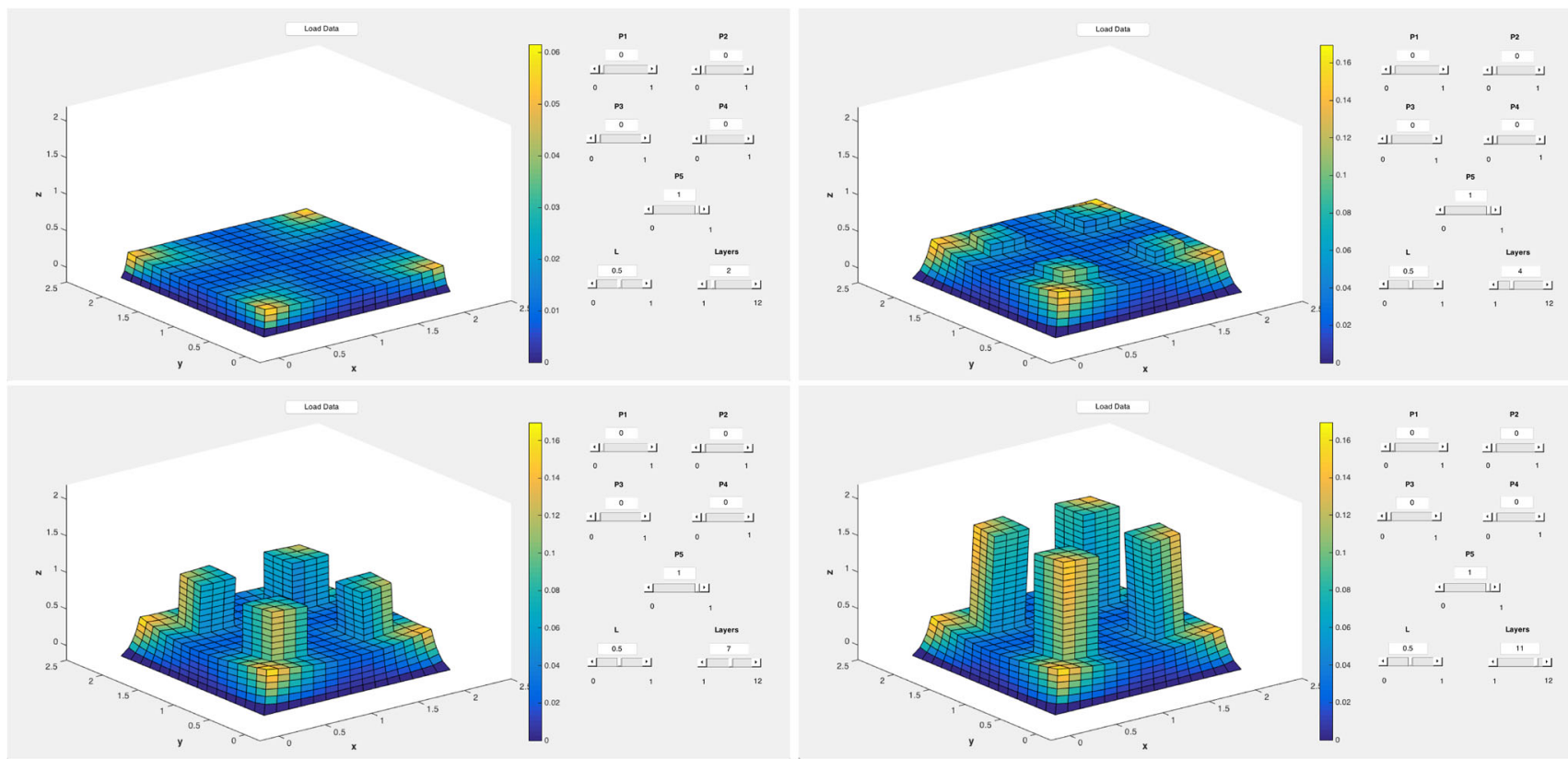

Fig. 10 Parametric distortions for $\mu_{1}=0, \mu_{2}=0, \mu_{3}=0, \mu_{4}=0, \mu_{5}=1, \lambda=L=0.5$ and: (top-left) layers $=2$; (top-right) layers $=4$; (bottom-left) layers $=7$ and (bottom-right) layers $=11$. Dimensions: $\times 10 \mathrm{~cm}$. Displacements in $\mathrm{cm}$

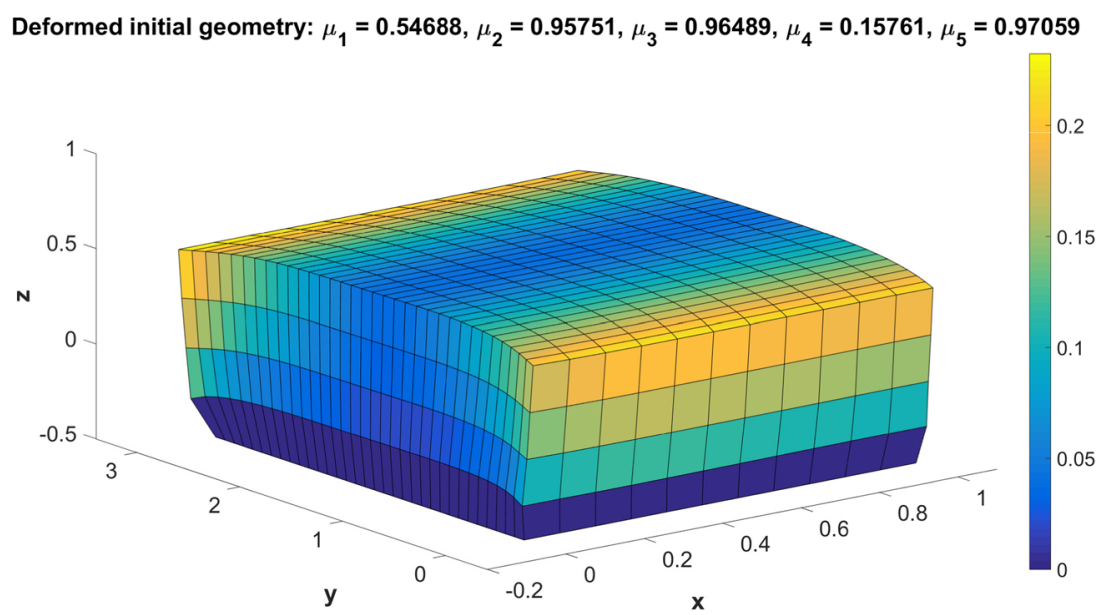

Deformed shape: $\mu_{1}=0.54688, \mu_{2}=0.95751, \mu_{3}=0.96489, \mu_{4}=0.15761, \mu_{5}=0.97059$

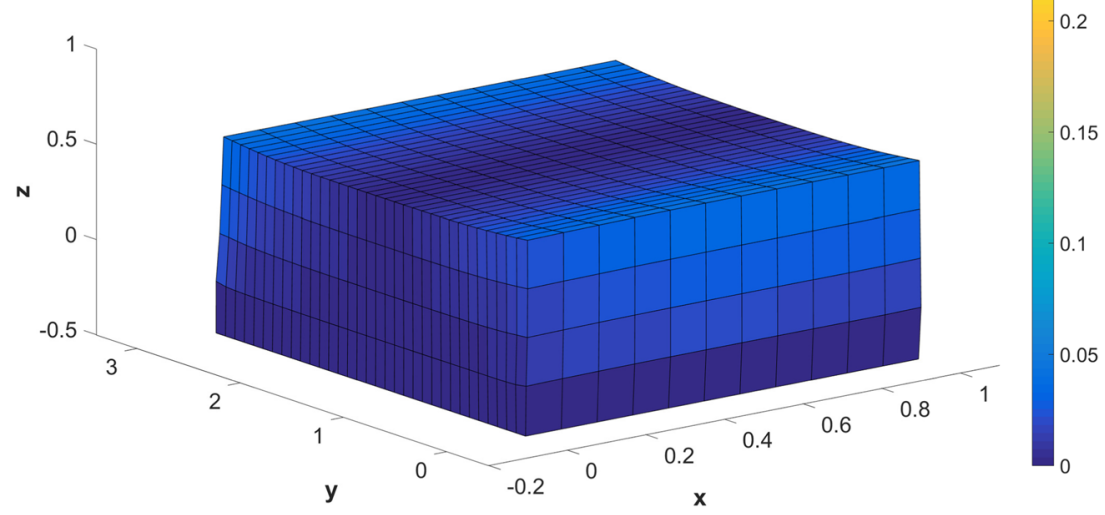

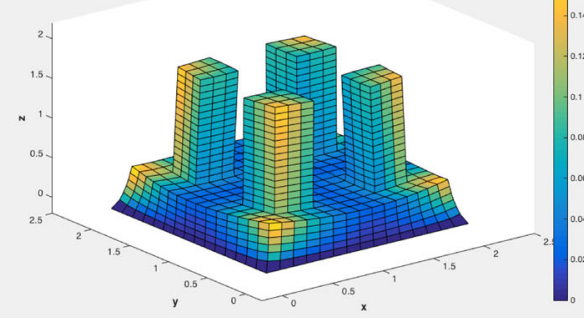

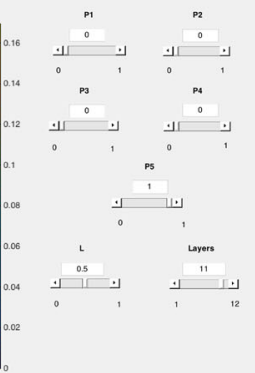




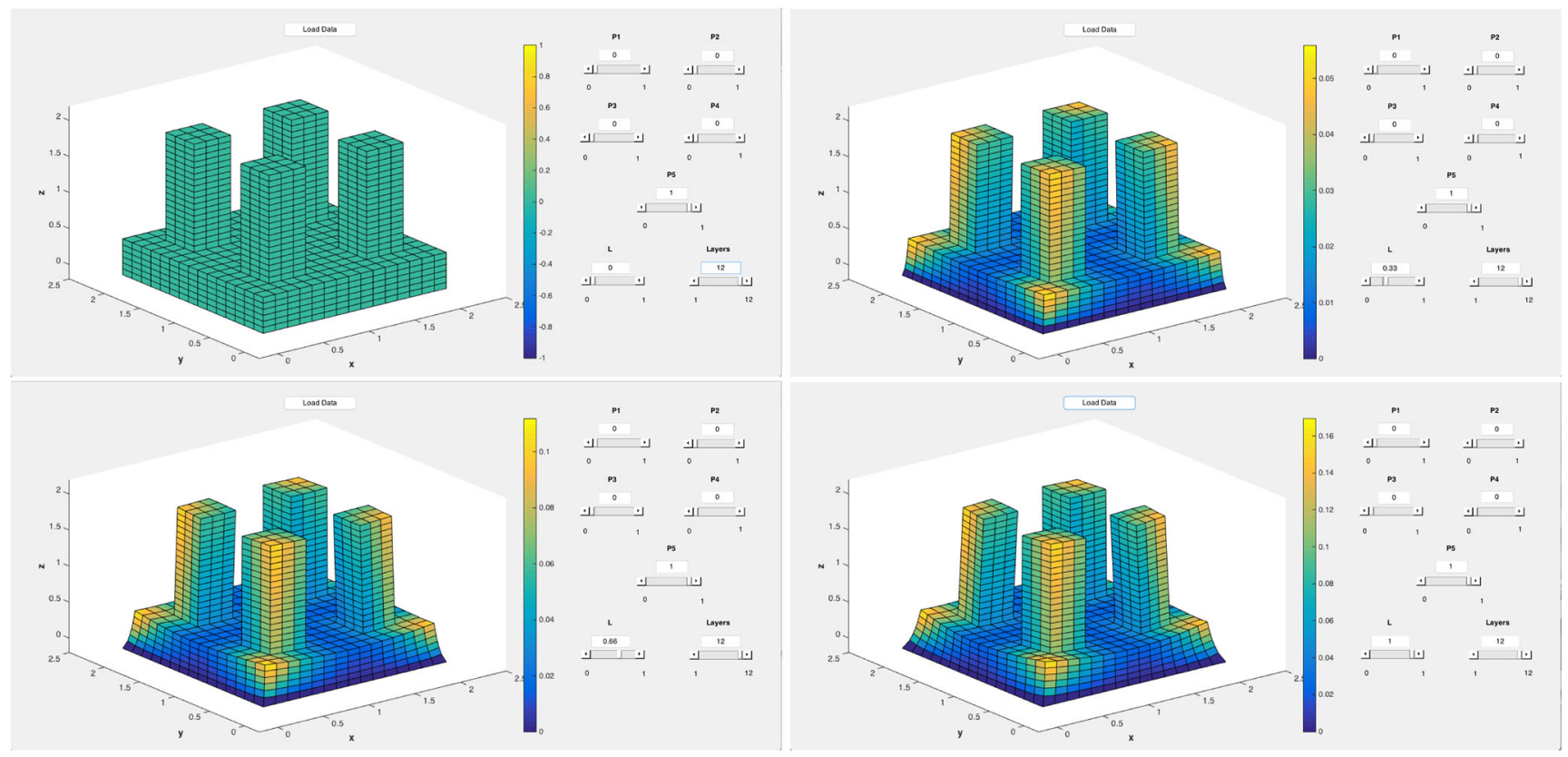

Fig. 11 Parametric distortions for $\mu_{1}=0, \mu_{2}=0, \mu_{3}=0, \mu_{4}=0, \mu_{5}=1$, layers $=12$ and: (top-left) $L=\lambda=0$; (top-right) $L=\lambda=0.33$; (bottom-left) $L=\lambda=0.66$ and (bottom-right) $L=\lambda=1$. Dimensions: $\times 10 \mathrm{~cm}$. Displacements in $\mathrm{cm}$

Fig. 12 Distorted final geometry for the indicated parameters defining the process trajectory. Dimensions: $\times 10 \mathrm{~cm}$. Displacements in $\mathrm{cm}$

Fig. 13 Compensated geometry. Dimensions: $\times 10 \mathrm{~cm}$. Displacements in $\mathrm{cm}$
Deformed shape: $\mu_{1}=0.3171, \mu_{2}=0.95022, \mu_{3}=0.034446, \mu_{4}=0.43874, \mu_{5}=0.38156$
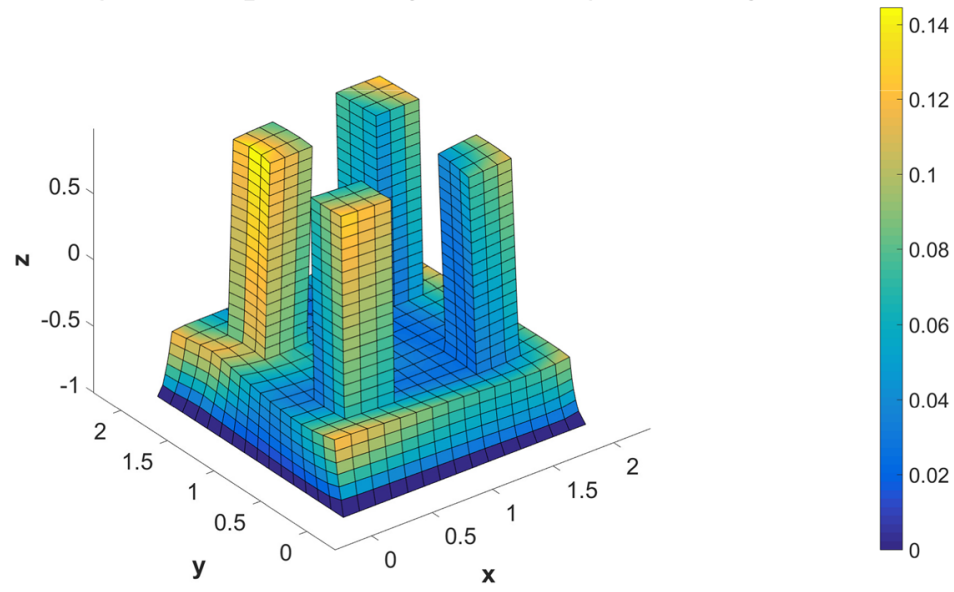

Deformed initial geometry: $\mu_{1}=0.3171, \mu_{2}=0.95022, \mu_{3}=0.034446, \mu_{4}=0.43874, \mu_{5}=0.38156$
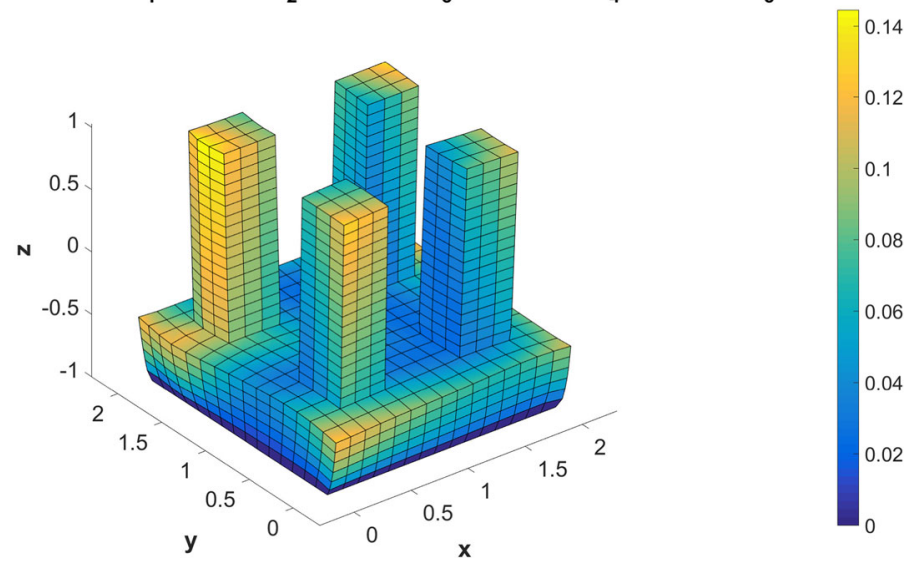
For that purpose we proceed from the parametric trajectories expression

$\phi\left(x, y, \mu_{1}, \cdots, \mu_{5}\right)=\sum_{i=1}^{5} \mu_{i} \phi^{i}(x, y)$,

whose normalized gradient defines the normal direction to the trajectory, according to

$\boldsymbol{n}=\frac{\nabla \phi}{\|\nabla \phi\|}$

with

$\nabla \phi\left(x, y, \mu_{1}, \cdots, \mu_{5}\right)=\sum_{i=1}^{5} \mu_{i} \nabla \phi^{i}(x, y)$,

where the gradient of solutions $\phi^{i}(x, t)$ can be computed offline.

Now, the tangent vector $\boldsymbol{t}$ comes from the normality condition $\boldsymbol{t} \cdot \boldsymbol{n}=0$. These expressions allow determining the parametric expression of the rotation tensor and from it, the one of the shrinkage tensor, by using the standard PGD technology even if the computation could be perfectly performed online in real-time without requiring precomputing a parametric expression of expression (11).

Now, the virtual work principle, assuming without loss of generality small displacements and strains, writes

$\int_{\Omega} \boldsymbol{\epsilon}^{*}:\left(\boldsymbol{\sigma}-\boldsymbol{\sigma}_{0}\right) d \boldsymbol{x}=0$,

where without loss of generality surface and volume forces are neglected. This expression applies for any kinematically admissible deformation $\epsilon^{*}$, i.e. related to test displacements vanishing in the part of the boundary of $\Omega$ where displacements are prescribed, in general the bottom basis of the part.

It can be noticed from Eq. (16) that shrinkage acts as a volume force and consequently in the resulting linear system (after discretization) it appears at the right-hand member, that can be expressed using the most usual notation as

$\mathbf{K} \mathbf{U}=\mathbf{F}\left(\mu_{1}, \cdots, \mu_{5}\right)$,

that as soon as the problem is linear, $\mathbf{K}^{-1}$ can be computed and used for evaluating online the parametric displacements according to

$\mathbf{U}\left(\mu_{1}, \cdots, \mu_{5}\right)=\mathbf{K}^{-1} \mathbf{F}\left(\mu_{1}, \cdots, \mu_{5}\right)$.

At this point it is important to make two observations:

- When considering nonlinear behaviors, in particular large strain / displacements, the best option consists of applying directly the PGD rationale for computing the parametric displacement field for each intermediate configuration, i.e. number of deposited layers, or applying the procedure just described operating at the discrete level (Eqs. 17 and 18) but decomposing the stiffness matrix in a linear and nonlinear parts, i.e. $\mathbf{K}=$ $\mathbf{K}_{L}+\mathbf{K}_{N L}$ and proceeding until convergence within the iteration scheme

$$
\begin{aligned}
\mathbf{U}^{n}\left(\mu_{1}, \cdots, \mu_{5}\right)= & \mathbf{K}_{L}^{-1}\left(-\mathbf{K}_{N L}^{n-1} \mathbf{U}^{n-1}(\boldsymbol{\mu})\right. \\
& \left.+\mathbf{F}\left(\mu_{1}, \cdots, \mu_{5}\right)\right),
\end{aligned}
$$

with $\mathbf{U}^{n-1}(\boldsymbol{\mu})=\mathbf{U}^{n-1}\left(\mu_{1}, \cdots, \mu_{5}\right)$.

- In all cases the linear and nonlinear matrices must be evaluated for different stages of the process, that is, for different numbers of deposited layers. Thus, later, the number of layers will be introduced as an extra-parameter in the parametric solution.

Figure 6 depicts, for a given shrinkage intensity $\lambda$, for the final configuration and for the different scenarios considered in Fig. 3, where parameters $\mu_{1}, \cdots, \mu_{4}$ and $\mu_{5}=s(x, y)$ are noted by $P 1, \cdots, P 5$, the considered solid cuboid part distortion from its target geometry. This GUI application allows evaluating distortions associated to any possible trajectory in almost real-time.

\section{Distorsion compensation}

As soon as the distortion is evaluated for a choice of the parameters, one could expect that by applying that displacement field with opposite sign to the target configuration, the final geometry after shrinkage should almost correspond to the target geometry. For evaluating this simple compensation procedure we consider again the part addressed in the previous section and evaluate its distortion for the parameters indicated in Fig. 7. Now, by applying to the target geometry the opposite of the displacement field illustrated in Fig. 7, it results the geometry displayed in Fig. 8. Now, using the same process parameters, the distorted part associated with the target geometry shown in Fig. 8 results in the one displayed in Fig. 9.

In the just analyzed case the geometry ensured that the simplest compensation procedure works perfectly, however this simple procedure could fail in more complex geometries. In that case it is obvious that the fact of evaluating in real-time (almost instantaneously) the displacement could allow to implement more sophisticated compensation algorithms dialoging with our computational vademecum.

\section{Numerical results}

In this section we are considering a quite more complex geometry and introducing two new parameters into the parametric solution (computational vademecum): (i) the 
Fig. 14 Distortion applying on the compensated geometry.

Dimensions: $\times 10 \mathrm{~cm}$

Displacements in $\mathrm{cm}$

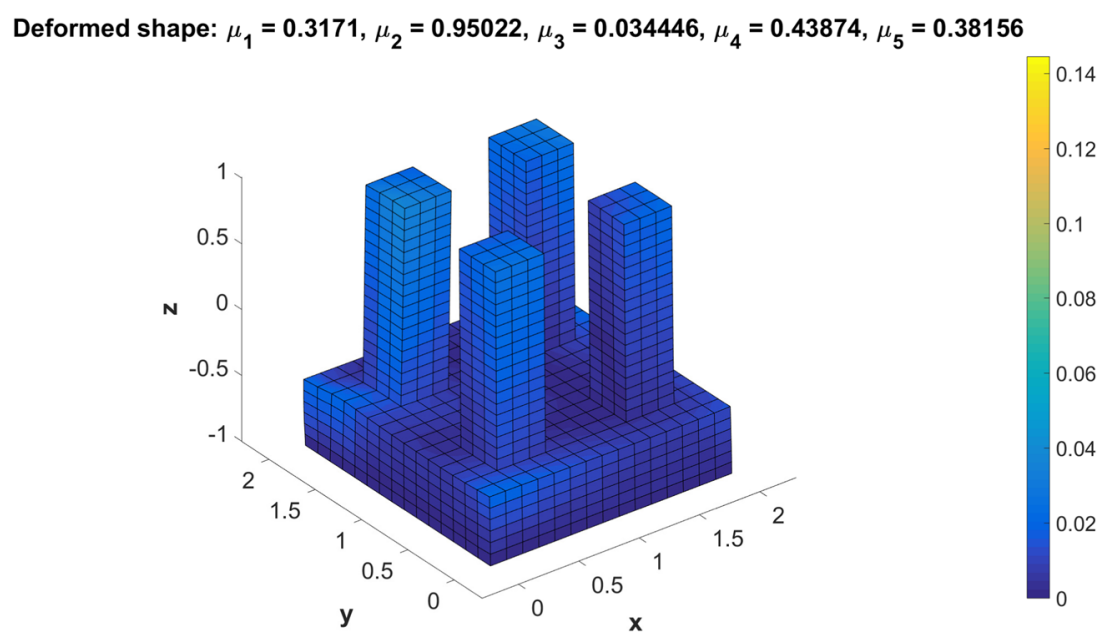

one related to the thermal shrinkage intensity $\lambda$ (noted in the GUI application shown later by $L$ ) and (ii) the process progression, that is, the number of deposited layers.

Figure 10 depicts the deformed geometry for four different process stages where different number of layers were deposited. Here the only parameter that varies is the number of layers. It can be noticed that the greater is the number of layers the higher is the distortion. Figure 11 shows the effect of the thermal shrinkage intensity, a certain value multiplied by parameter $L \equiv \lambda$, from a null value for with distortions vanish to its highest value $L=1$ exhibiting the largest distortions.

\section{Geometry compensation}

For evaluating in the present case study the compensation procedure we evaluate its distortion for the parameters indicated in Fig. 12. Now, by applying to the target geometry the opposite of the displacement field illustrated in Fig. 12, it results the geometry displayed in Fig. 13. Now, using te same process parameters, the distorted part
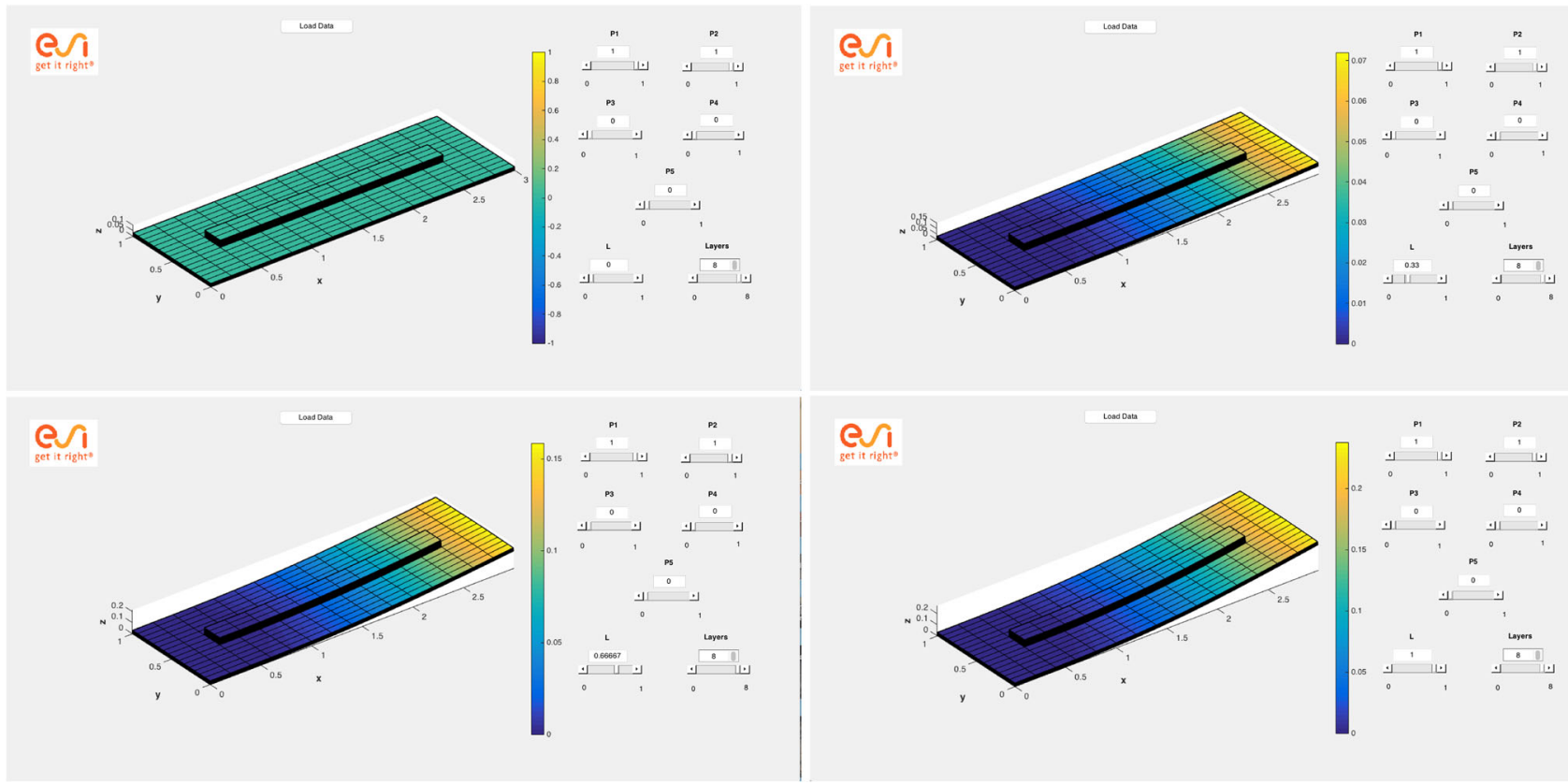

Fig. 15 Parametric distortions for $\mu_{1}=1, \mu_{2}=1, \mu_{3}=0, \mu_{4}=0, \mu_{5}=0$, layers $=8$ and: (top-left) $L=\lambda=0$; (top-right) $L=\lambda=0.33$; (bottom-left) $L=\lambda=0.66$ and (bottom-right) $L=\lambda=1$. Dimensions: $\times 10 \mathrm{~cm}$. Displacements: $\times 10 \mathrm{~cm}$ 

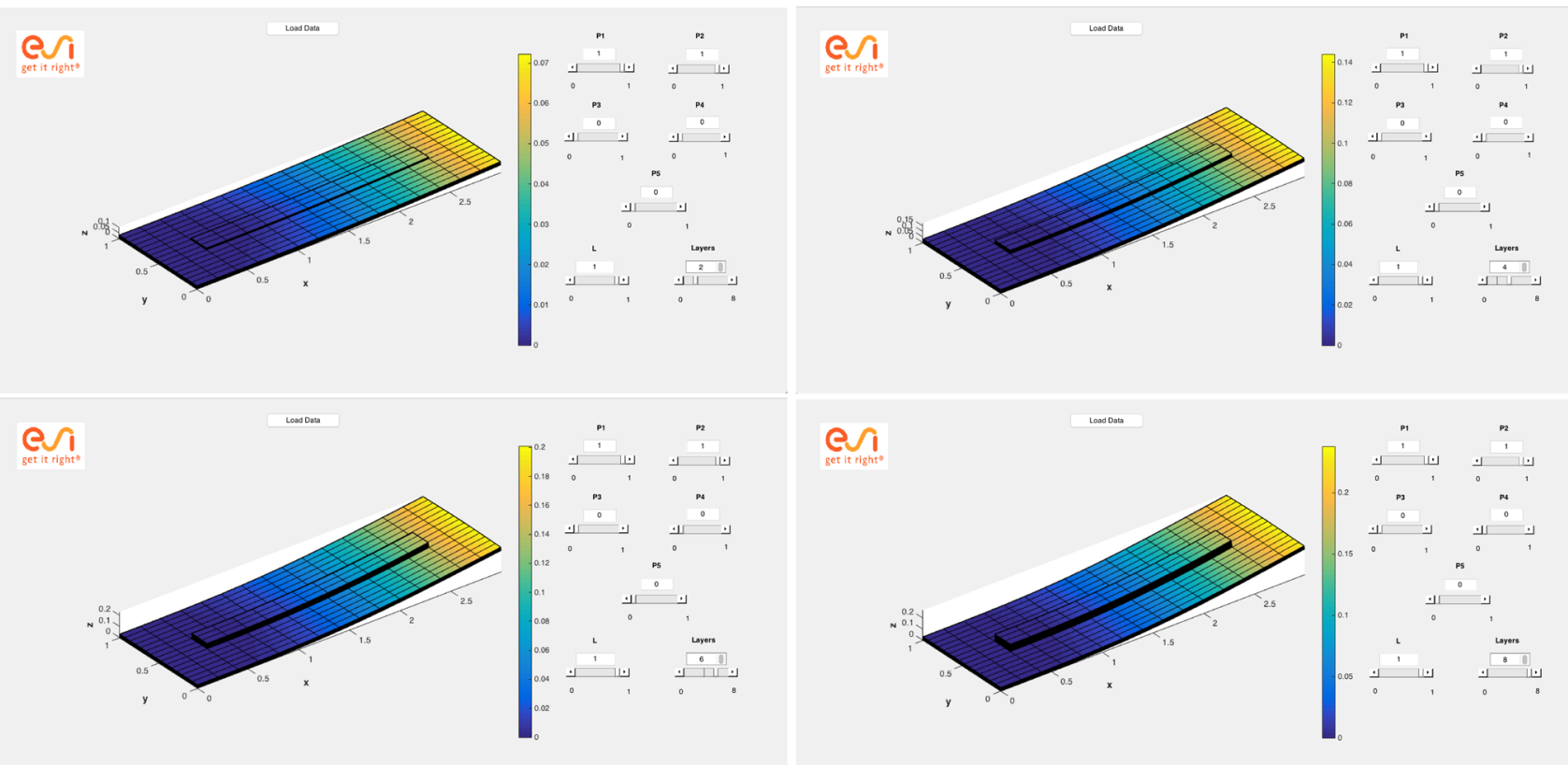

Fig. 16 Parametric distortions for $\mu_{1}=1, \mu_{2}=1, \mu_{3}=0, \mu_{4}=0, \mu_{5}=0, \lambda=L=1$ and: (top-left) layers $=2$; (top-right) layers $=4$; (bottom-left) layers $=6$ and (bottom-right) layers $=8$. Dimensions: $\times 10 \mathrm{~cm}$. Displacements: $\times 10 \mathrm{~cm}$

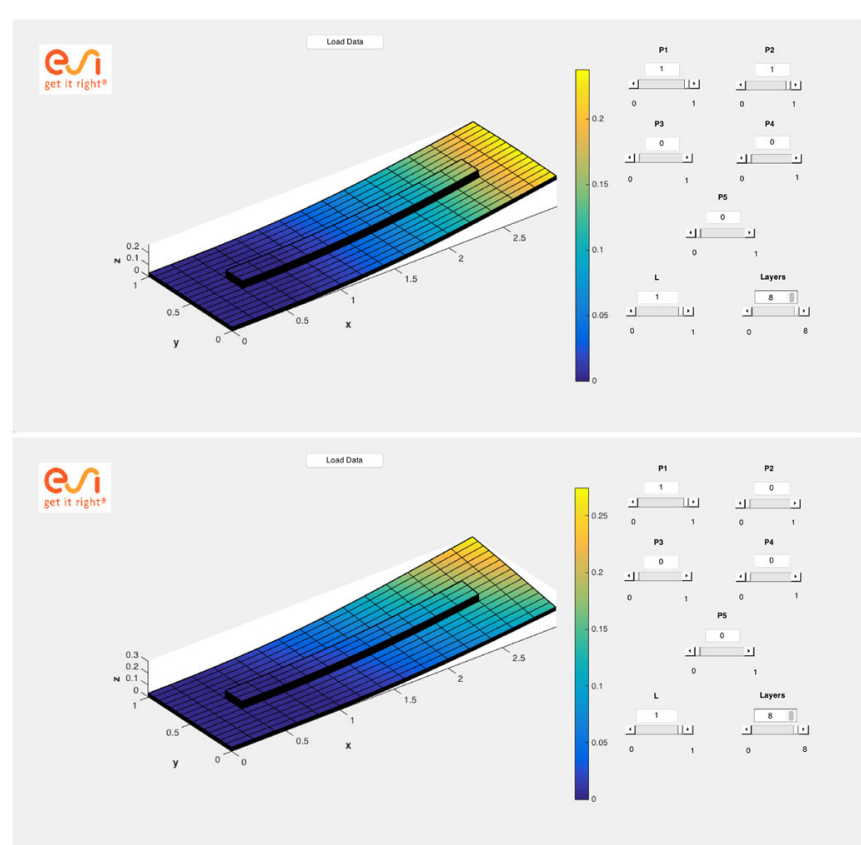

Fig. 17 Parametric distortions for layers $=8, \lambda=L=1$ and: (top-left) $\mu_{1}=1, \mu_{2}=1, \mu_{3}=0, \mu_{4}=0, \mu_{5}=0$; (topright) $\mu_{1}=1, \mu_{2}=0, \mu_{3}=0, \mu_{4}=0, \mu_{5}=0$; (bottom-left)

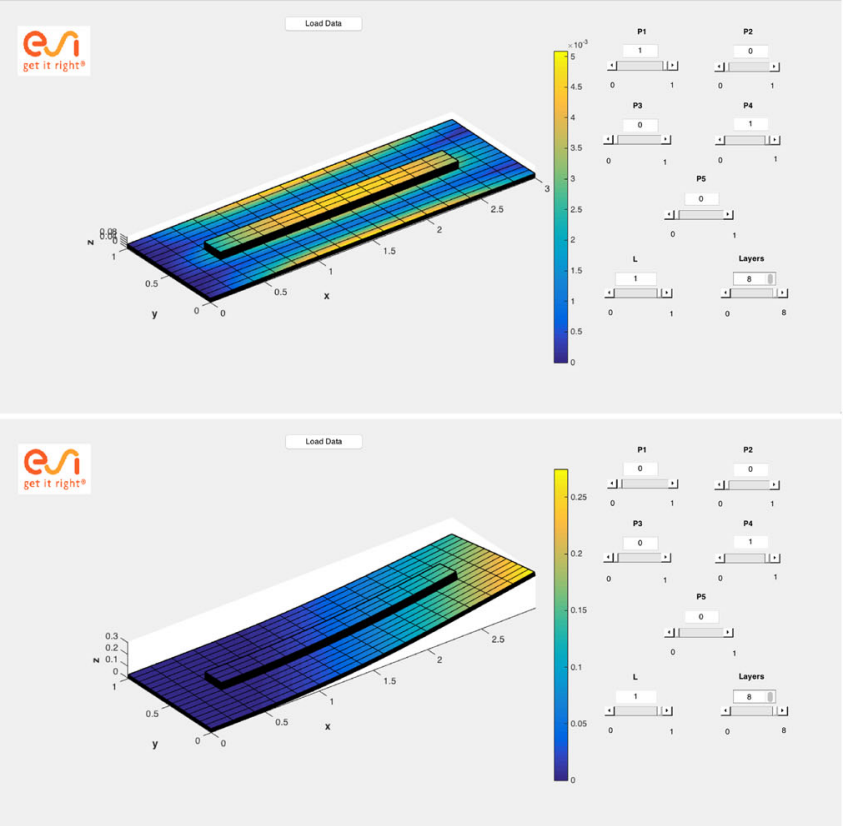

$\mu_{1}=0, \mu_{2}=0, \mu_{3}=0, \mu_{4}=0, \mu_{5}=0$ and (bottom-right) $\mu_{1}=0, \mu_{2}=0, \mu_{3}=0, \mu_{4}=1, \mu_{5}=0$. Dimensions: $\times 10 \mathrm{~cm}$. Displacements: $\times 10 \mathrm{~cm}$
Fig. 18 Experimental test (courtesy of J.Y. Hascoet at GeM Institute - Ecole Centrale de Nantes)

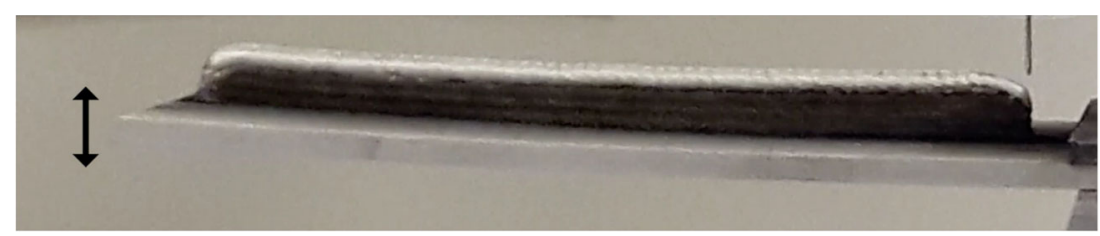


associated with the target geometry shown in Fig. 13 results in the one displayed in Fig. 14.

\section{Qualitative validation}

For a first qualitative validation we considered the deposition of a thin wall on a thin plate with a prescribed null displacements at one of its lateral bases. Figures 15, 16 and 17 depict the distorted structure for respectively different shrinkage intensities, number of deposited layers and process trajectories. Figure 18 shows the experimental result when the process is operated by using trajectories expressed by $\mu_{1}=\mu_{2}=1$ and $\mu_{3}=\mu_{4}=\mu_{5}=0$.

\section{Conclusions}

This paper presents a simplified parametric modeling of distortions occurring in parts manufactured by additive manufacturing. After having introduced a parametrization of the process trajectories as the iso-values of a parametrized partial differential equation, thermal shrinkage was expressed parametrically with respect to the process trajectory. Finally two new parameters were introduced, the first controlling the shrinkage intensity and the second the process progression, that is, the number of deposited layers.

The parametric solution was introduced into a GUI application able to evaluate the effect of any parameter in almost real-time. Moreover, parametric solutions open an appealing route for geometry compensation.

The numerical examples illustrate the effect of the different parameters and even if at present a quantitative validation requires additional efforts (it constitutes a work in progress), the first qualitative validation seems very promising.

In the present work the use model order reduction was limited at maximum, profiting the linearity of the trajectory description and the solution of the mechanical problem in both the linear and nonlinear setting. Thus, the solution accuracy is the one of the standard finite element discretization, without being impacted by reduced modeling issues. The works in progress consider a parametric modeling within the PGD framework, to consider the process as a parametric black box allowing higher flexibility and generality in what concerns the physics involved.

Acknowledgements This project has received funding from the European Union's Horizon 2020 research and innovation programme under the Marie Sklodowska-Curie grant agreement No. 675919. Authors also thank the partners of the SOFIA projet.

\section{Compliance with Ethical Standards}

Conflict of interests The authors declare that they have no conflict of interest.

Publisher's Note Springer Nature remains neutral with regard to jurisdictional claims in published maps and institutional affiliations.

\section{References}

1. Aguado JV, Bognet B, Canales D, Desmaison O, Boitout F, Chinesta F (2017) A reduced order modeling approach for fast thermo-mechanics simulation of additive layer manufacturing. ECCOMAS Conference on Simulation for Additive Manufacturing, Munich, Germany

2. Bur N, Joyot P, Ghnatios C et al (2016) Adv Model Simul Eng Sci 3:4. https://doi.org/10.1186/s40323-016-0056-x

3. Chen T, Zhang Y, Yuwen (2004) Numerical simulation of twodimensional melting and resolidification of a two-component metal powder layer in selective laser sintering process. Numerical Heat Transfer, Part A: Applications 46(7):633-649

4. Chinesta F, Ladeveze P, Cueto E (2011) A short review in model order reduction based on proper generalized decomposition. Arch Comput Methods Eng 18:395-404

5. Chinesta F, Leygue A, Bordeu F, Aguado JV, Cueto E, Gonzalez D, Alfaro I, Ammar A, Huerta A (2013) PGD-based computational vademecum for efficient design, optimization and control. Arch Comput Methods Eng 20:31-59

6. Chinesta F, Keunings R, Leygue A (2014) The proper generalized decomposition for advanced numerical simulations. A primer. Springerbriefs, Springer

7. Chinesta F, Ladeveze P (eds) (2014) Separated Representations and PGD Based Model Reduction: Fundamentals and Applications. CISM-Springer, Berlin

8. Chinesta F, Leygue A, Bognet B, Ghnatios C, Poulhaon F, Bordeu F, Barasinski A, Poitou A, Chatel S, Maison-Le-Poec S (2014) First steps towards an advanced simulation of composites manufacturing by automated tape placement. Int J Mater Form 7:81-92

9. Chinesta F, Huerta A, Rozza G, Willcox K (2015). In: Stein E, de Borst R, Hughes T (eds) Model order reduction. Chapter in the encyclopedia of computational mechanics, 2nd edn. Wiley, New York

10. Chiumenti M, Cervera M, Salmi A, Agelet de Saracibar C, Dialami N, Matsui K (2010) Finite element modeling of multipass welding and shaped metal deposition processes. Comput Methods Appl Mech Eng 199(37):2343-2359

11. Chiumenti M, Lin X, Cervera M, Lei W, Zheng Y, Huang W (2017) Numerical simulation and experimental calibration of Additive Manufacturing by blown powder technology. Part I: thermal analysis. Rapid Prototyping Journal. (In press)

12. Dai D, Gu D (2014) Thermal behavior and densification mechanism during selective laser melting of copper matrix composites: Simulation and experiments. Mater Des 55:482491

13. Ding J, Colegrove P, Mehnen J, Sequeira Almeida PM, Wang F, Williams S (2011) Thermomechanical analysis of wire and arc additive layer manufacturing process on large multi-layer parts. Comput Mater Sci 50:3315-3322 
14. Dong L, Makradi A, Ahzi S, Remond Y (2009) Threedimensional transient finite element analysis of the selective laser sintering process. J Mater Process Technol 209(2):700706

15. Foteinopoulos $\mathrm{P}$, Papacharalampopoulos A, Stavropoulos $\mathrm{P}$ (2018) On thermal modeling of additive manufacturing processes. CIRP J Manuf Sci Technol 20:66-83

16. Heigel JC, Michaleris P, Reutzel EW (2015) Thermo-mechanical model development and validation of directed energy deposition additive manufacturing of Ti-6Al-4V. Addit Manuf 5:919

17. Khairallah SA, Anderson AT, Rubenchik A, King WE (2016) Laser powder-bed fusion additive manufacturing: Physics of complex melt flow and formation mechanisms of pores, spatter and denudation zones. Acta Mater 108:3645

18. Korner C, Attar E, Heinl P (2011) Mesoscopic simulation of selective beam melting processes. J Mater Process Technol 211(6):978-987

19. Korner C, Bauereis A, Attar E (2013) Fundamental consolidation mechanisms during selective beam melting of powders. Model Simul Mater Sci Eng 21(8):085011

20. Kolossov S, Boillat E, Glardon R, Fischer P, Locher M (2004) 3D FE simulation for temperature evolution in the selective laser sintering process. Int J Mach Tools Manuf 44(2):117123
21. Kovaleva I, Kovalev O, Smurov I (2014) Model of heat and mass transfer in random packing layer of powder particles in selective laser melting. Phys Procedia 56:400-410

22. Labudovic M, Hu D, Kovacevic R (2003) A three dimensional model for direct laser metal powder deposition and rapid prototyping. J Mater Sci 38:35-49

23. Loh L, Chua C, Yeong W, Song J, Mapar M, Sing S, Liu Z, Zhang D (2015) Numerical investigation and an effective modelling on the selective laser melting (SLM) process with aluminium alloy 6061. Int J Heat Mass Transfer 80:288-300

24. Li Y, Gu D (2014) Parametric analysis of thermal behavior during selective laser melting additive manufacturing of aluminum alloy powder. Mater Des 63:856-867

25. Marimuthu S, Clark D, Allen J, Kamara AM, Mativenga P, Li L, Scudamore R (2013) Finite element modelling of substrate thermal distortion in direct laser additive manufacture of an aeroengine component. Proc Inst Mech Eng C J Mech Eng Sci 227(9):1987-1999

26. Zekovic S, Dwivedi R, Kovacevic R (2005) Thermo-structural finite element analysis of direct laser metal deposited thin-walled structures. In: 16th Solid Freeform Fabrication Symposium, SFF 2005, pp 338-355

27. Zeng K, Pal D, Patil N, Stucker BE (2013) A new dynamic mesh method applied to the simulation of selective laser melting. Proceedings of the Solid Freeform Fabrication Symposium, Austin, TX Aug. 12-14, pp 549-559 